\title{
Parásitos intestinales y factores de riesgo en escolares de una institución educativa rural de Tunja (Colombia) en el año 2015
}

\author{
Intestinal parasites and risk factors in schoolchildren \\ of a rural school from Tunja (Colombia) in 2015
}

\begin{abstract}
Ana Y. Rodríguez-Sáenz MSc ${ }^{1}$, Sergio A. Mozo-Pacheco MD', Lisandro E. Mejía-Peñuela $M D^{2}$
\end{abstract}

Introducción: una de las enfermedades infecciosas más común es el parasitismo intestinal, encontrándose ampliamente distribuido a nivel mundial, especialmente en las personas más vulnerables. objetivo: determinar la prevalencia de parásitos intestinales y los factores de riesgo en escolares de una institución educativa rural de la ciudad de Tunja (Colombia). Materiales y métodos: se llevó a cabo una investigación de tipo descriptivo transversal, en la cual aceptaron participar 89 niños con previa firma del asentimiento y consentimiento informado por parte de sus padres. Se recolectaron muestras de heces fecales de cada uno de los niños y se les practicó examen coprológico y análisis por medio de la técnica de concentración formol-éter. Los factores de riesgo se evaluaron con la ayuda de una encuesta que contempló preguntas sobre los hábitos higiénico-sanitarios y las características de las viviendas. Resultados: la prevalencia general de parásitos intestinales fue del $74,2 \%$, siendo Entamoeba histolytica/dispar (90,9\%), Endolimax nana (66,7\%) y Entamoeba coli $(60,6 \%)$ las especies más frecuentes. Dentro de los factores de riesgo se destacan la medicación en casa, el contacto con animales, la disposición de aguas residuales al aire libre, jugar con tierra y caminar descalzos. No se encontró relación estadísticamente significativa entre los factores de riesgo y el parasitismo intestinal. Conclusiones: la proporción de parásitos intestinales en los escolares estudiados fue alta $(74,2 \%)$, con predominio de los protozoos y los parásitos no patógenos. Es importante continuar incrementando las actividades educativas encaminadas a prevenir el parasitismo intestinal.

Palabras clave: niño, parasitosis intestinales, factores de riesgo.

Rodríguez-Sáenz AY, Mozo-Pacheco SA, Mejía-Peñuela LE. Parásitos intestinales y factores de riesgo en escolares de una institución educativa rural de Tunja (Colombia) en el año 2015. Medicina \& Laboratorio 2017; 23: 159-170.

${ }^{1}$ Bacterióloga, MSc en Microbiología. Docente, Escuela de Medicina, Universidad Pedagógica y Tecnológica de Colombia (UPTC). Tunja, Colombia. Correspondencia: Calle 24 \#

5-63. Correo electrónico: anayervid.rodriguez@uptc.edu.co

${ }^{2}$ Estudiantes de Medicina, Universidad Pedagógica y Tecnológica de Colombia (UPTC).

Tunja, Colombia.

Conflicto de intereses: los autores declaran que no tienen conflicto de intereses

Medicina \& Laboratorio 2017; 23: 159-170

Módulo 19 (Investigación), número 54. Editora Médica Colombiana S.A. $2017^{\circ}$

Recibido el 23 de febrero de 2017; aceptado el 17 de abril de 2017 
os parásitos intestinales son organismos infecciosos que se alojan en el tracto gastrointestinal de distintos seres vivos. Desde las antiguas civilizaciones, aquellos denominados parásitos patógenos han causado enfermedades en los seres humanos, los cuales representan uno de sus principales hospederos [1-4]. Los parásitos intestinales, ya sean unicelulares (protozoos) o pluricelulares (helmintos), producen una amplia gama de cuadros clínicos, sintomatología y afecciones nutricionales, lo que representa un importante problema de salud pública $[5,6]$.

La Organización Mundial de la Salud (OMS) considera que aproximadamente el $24 \%$ de la población mundial (1.500.000.000 de personas) está infectada por parásitos intestinales transmitidos por el suelo (geohelmintos), con mayor prevalencia en zonas geográficas del África subsahariana, América, China y Asia oriental, donde además la población infantil es más susceptible a la infección por parásitos intestinales, debido a la exposición constante a los factores de riesgo, tales como no usar calzado, jugar con tierra, consumir agua no potable y disponer de forma inadecuada las basuras [7]. Según la encuesta nacional de parasitismo intestinal en la población escolar, fase II del Ministerio de Salud y Protección Social de Colombia, en el país existe un alto predominio de parásitos intestinales $(62,4 \%$ protozoos y $56,2 \%$ geohelmintos) en la población escolar, lo que genera otros problemas como la anemia, la malnutrición, el deterioro del crecimiento y el desarrollo infantil, la memoria, el razonamiento y el coeficiente intelectual [8].

Los factores epidemiológicos que favorecen la propagación de parásitos instestinales incluyen: a) la contaminación fecal debida a malos hábitos de higiene como el inadecuado lavado de manos, aseo personal y lavado de los alimentos, b) las condiciones ambientales asociadas generalmente a las características geográficas y climáticas de las zonas tropicales o subtropicales y c) la vida rural, ya que los niveles de pobreza condicionan a la falta de educación y la carencia de condiciones higiénicosanitarias adecuadas, como disposición de agua potable, servicio sanitario, alcantarillado y energía eléctrica, que, en conjunto con las costumbres alimenticias y culturales, llevan al consumo de alimentos mal cocidos, a permanecer en contacto cercano con animales y a andar descalzos, lo que finalmente favorece la transmisión de los parásitos [9-12].

Estudios previos sobre la implementación de estrategias de educación en salud han demostrado, por medio de experiencias exitosas, el control integral de las parasitosis intestinales, lo cual ratifica la importancia de generar, desde las entidades gubernamentales, académicas y el personal de la salud, campañas de prevención con adecuadas sesiones educativas que incentiven en las comunidades los buenos hábitos de higiene y alimentación para disminuir la tasa de infecciones parasitarias y contribuir a reducir sus consecuencias $[13,14]$.

Los niños en edad escolar son considerados epidemiológicamente como una población susceptible a las infecciones por parásitos intestinales [5]. Por lo anterior, el presente estudio tuvo como objetivo determinar la frecuencia de parásitos intestinales y los factores de riesgo asociados en escolares de una institución educativa rural de la ciudad de Tunja, Colombia. 


\section{Materiales y métodos}

\section{Población estudiada}

Se realizó un estudio descriptivo transversal en una institución educativa rural de Tunja (Colombia). Se tomó como universo el total de los escolares de preescolar y básica primaria $(n=90)$. La muestra fue condicionada a la participación voluntaria y 89 niños de los grados preescolar a quinto fueron autorizados por sus padres para participar en el estudio, quedando así la muestra conformada.

\section{Aspectos éticos}

La investigación siguió las normas éticas para trabajos en la comunidad, tratadas en la resolución 008430 de 1993 del Ministerio de Salud de Colombia [15]. También fue aprobada previamente por el comité de ética de la Universidad Pedagógica y Tecnológica de Colombia (UPTC) de Tunja (Colombia). Previo al estudio se dio información del proyecto a los escolares, padres de familia y a los profesores de la institución educativa.

\section{Estudio parasitológico}

Se recolectaron muestras de heces fecales en un frasco estéril con solución de formalina al $5 \%$ y se transportaron al laboratorio de Microbiología de la Facultad de Ciencias de la Salud de la Universidad Pedagógica y Tecnológica de Colombia (UPTC) en la ciudad de Tunja (Colombia), donde se procesaron para examen coprológico con lugol al $1 \%$ y solución salina al $0,83 \%$ y y para la técnica de concentración formol-éter según Richie [9].

Finalizado el procesamiento de las muestras se impartieron sesiones educativas sobre la prevención, se informaron los resultados obtenidos a los profesores, niños y padres de familia $y$, posteriormente, un médico impar- tió tratamiento antiparasitario a los escolares según el caso (protozoos o helmintos).

\section{Recolección de información}

Se aplicó a los padres y niños una encuesta sobre los factores de riesgo que contribuyen a la adquisición de parásitos intestinales, la cual incluía preguntas sobre la procedencia del agua para consumo, la presencia de sanitario en las viviendas, el tipo de piso en las viviendas, la disposición de aguas residuales, la eliminación de basuras, la presencia de servicios públicos, el lavado de manos antes de comer y después de defecar, el lavado de frutas y verduras antes de consumirlos, caminar descalzos, el contacto con animales, la automedicación y la asistencia al médico.

\section{Análisis estadístico}

Los datos de las variables obtenidas de las encuestas de factores de riesgo y parásitos intestinales fueron organizados en tablas electrónicas en el programa Microsoft Excel $^{\circledR}$ (Microsoft, Washington, Estados Unidos), posteriormente se realizó un análisis descriptivo de las variables mediante el programa estadístico SPSS versión 21 (IBM, Nueva York, Estados Unidos) por medio de medidas de tendencia central y descriptivas como la prueba de chi-cuadrado para analizar la significancia entre las variables.

\section{Resultados}

Se evaluaron, durante los meses de agosto y noviembre, 89 niños en edades entre los 5 y los 13 años, de los cuales 46 (51,7\%) fueron niñas y $43(48,3 \%)$ niños. La prevalencia general de parásitos intestinales en los 89 niños muestreados fue del $74,2 \%$ ( $n=66)$; en el $25,8 \%(23 / 89)$ de los niños no se obser- 
Tabla 1. Prevalencia de parásitos intestinales según grado escolar en una institución educativa rural de Tunja

\begin{tabular}{lllll}
\hline \multirow{1}{*}{ Grado } & \multicolumn{5}{c}{ Parasitismo } \\
& \multicolumn{2}{c}{ Ausente } & \multicolumn{2}{c}{ Presente } \\
& N. ${ }^{\circ}$ & \% & N. $^{\circ}$ & \% \\
\hline Preescolar & 1 & 1,1 & 10 & 11,2 \\
Primero & 4 & 4,5 & 9 & 10,1 \\
Segundo & 3 & 3,4 & 11 & 12,4 \\
Tercero & 5 & 5,6 & 19 & 21,3 \\
Cuarto & 4 & 4,5 & 7 & 7,9 \\
Quinto & 6 & 6,7 & 10 & 11,2 \\
Total & 23 & 25,8 & 66 & 74,2 \\
\hline
\end{tabular}

varon parásitos intestinales. En cuanto el grado escolar, el grado tercero fue el que presentó mayor número de casos positivos para parásitos intestinales $(21,3 \%, 19 / 89)$, seguido por el grado segundo $(12,4 \%$, $11 / 89)$, mientras que el cuarto grado fue el que obtuvo el menor número $(7,9 \%, 7 / 89)$ (véase tabla 1). No se hallaron diferencias significativas entre géneros y entre cada uno de los grados escolares referente a los parásitos encontrados.

Del total de niños parasitados en el $4,6 \%$ (3/66) se encontró un solo parásito, el $24,2 \%(16 / 66)$ estaban biparasitados y el $71,2 \%(47 / 66)$ poliparasitados, en los que se hallaron entre tres y seis especies de parásitos. Las especies parasitarias más identificadas fueron protozoos en el $100 \%$ (66/66) de niños parasitados, en comparación con los helmintos, los cuales se encontraron en el $19,7 \%$ (13/66). La prevalencia de parásitos no patógenos fue ligeramente superior, con una frecuencia del $95,5 \%(63 / 66)$, en relación con los parásitos patógenos que fue del 92,4\% (61/66). Entamoeba histolytica/ dispar (90,9\%; 60/66) fue la especie encontrada en mayor frecuencia, seguida de $E n$ dolimax nana $(66,7 \%, 44 / 66)$ y Entamoeba
Tabla 2. Parásitos intestinales en 66 de los 89 escolares de una institución educativa rural de Tunja, Colombia

\begin{tabular}{lll}
\hline Parásito & \multicolumn{2}{c}{ Prevalencia } \\
& N. & \% \\
\hline Entamoeba histolytica/dispar & 60 & 90,9 \\
Endolimax nana & 44 & 66,7 \\
Entamoeba coli & 40 & 60,6 \\
Blastocystis hominis & 20 & 30,3 \\
Ascaris lumbricoides & 10 & 15,1 \\
lodamoeba butschlii & 7 & 10,6 \\
Giardia intestinalis & 4 & 6,0 \\
Ancylostoma duodenale/Necator & 3 & 4,5 \\
americanus (uncinarias) & & \\
Trichuris trichiura & 1 & 1,5 \\
Chilomastix mesnili & 1 & 1,5 \\
\hline
\end{tabular}

coli $(60,6 \%, 40 / 66)$. En la tabla 2 se presentan los parásitos intestinales identificados en los 66 niños con resultados positivos.

Los resultados obtenidos de las encuestas epidemiológicas para la detección de factores de riesgo revelaron que en las viviendas del $83,1 \%$ (74/89) de los escolares el agua para consumo procede del acueducto y en el $16,9 \%$ (15/89) de pozo; un 73,0\% (65/89) de las madres hierve el agua para el consumo, un $74,2 \%(66 / 89)$ de los escolares se lava las manos antes de comer y luego de defecar y el $82,0 \%$ (73/89) lava las frutas y verduras antes de comerlas. Por otra parte, el 53,9\% (48/89) camina descalzo, el $56,2 \%(50 / 89)$ tiene contacto con la tierra y el $82,0 \%(73 / 89)$ con animales. En cuanto al material del piso de la vivienda el $38,2 \%$ (34/89) es de concreto y el 2,2\% (2/89) de tierra, y el 96,6\% (86/89) tiene un sistema adecuado de eliminación de excretas (poseen sanitario). El principal servicio público con el que cuentan es el agua en el $96,6 \%$ (86/89) de las viviendas, pero en el $3,4 \%$ (3/89) no poseen servicios públicos. 
En la mayor parte de las viviendas $(76,4 \%$, $68 / 89$ ) realizan la disposición de aguas residuales al aire libre y en las restantes $(23,6 \%$, 21/89) en fosas. La disposición de basuras la realizan al aire libre en el 37,1\% (33/89) de las viviendas; el $47,2 \%(42 / 89)$ las quema, el $12,4 \%(11 / 89)$ se deshace de ella por medio de empresa recolectora y el 3,4\% (3/89) la destina al reciclaje. Finalmente, el $71,9 \%$ (64/89) de los escolares es llevado a los servicios médicos cuando están enfermos y el $92,1 \%(82 / 89)$ recibe medicación en casa con remedios caseros. Los resultados de la encuesta relacionados con el parasitismo se presentan en la tabla $\mathbf{3}$.

Al realizar el análisis bivariado no se encontró asociación estadística significativa entre la presencia de parásitos intestinales o el tipo de parásito con los factores de riesgo para la adquisición de los mismos (chi-cuadrado $=0,553$; valor $p=0,063$ ) (véase tabla 3). En cuanto al género, aun cuando no se observaron diferencias estadísticamente significativas (chi-cuadrado $=$ 0,360 ; valor $p=0,097$ ), las niñas mostraron una mayor tendencia parasitaria que los niños; especialmente para las especies de Entamoeba histolytica/dispar, encontrada en el 55,0\% (49/89), y Endolimax nana con un $56,8 \%(50 / 89)$.

Los escolares recibieron tratamiento antiparasitario formulado por un médico, según el reporte de los resultados del examen coprológico y el análisis por método de concentración de Richie. Las sesiones educativas estuvieron encaminadas a prevenir el parasitismo intestinal con el desarrollo de una enseñanza de la higiene personal y la creación de hábitos de salud en los niños, extendiendo dicha enseñanza a los padres de familia.

\section{Discusión}

Los parásitos intestinales son los agentes infecciosos más comunes en los humanos, encontrándose ampliamente diseminados alrededor del mundo; sin embargo, los países tropicales y subtropicales reúnen las características geográficas y climatológicas que favorecen el predominio de la mayoría de estos organismos. Teniendo en cuenta que las parasitosis pueden ser prevenidas y controladas con acciones sanitarias específicas [14] se determinó la prevalencia de parásitos intestinales en los escolares de la institución educativa de estudio, la cual fue del $74,2 \%$, muy similar a la obtenida en otras investigaciones nacionales como la de Cardona-Arias y Bedoya-Urrego [16], donde fue del $74,4 \%$, lo que refleja la susceptibilidad a las infecciones parasitarias intestinales en la población escolar. No obstante, fue inferior a las encontradas en estudios realizados tanto en el ámbito nacional como internacional $(74,2 \%$ frente a $92,3 \%, 80 \%$, $78 \%$ y $87 \%)[5,8,10,11]$.

La frecuencia de protozoos (100\%) fue mucho mayor a la de helmintos $(19,7 \%)$, lo que concuerda con la encuesta nacional de parasitismo en población escolar 2012-2013, donde el porcentaje de protozoos $(62,4 \%)$ fue mayor a la de helmintos $(56,2 \%)$ [8], pero difiere de estudios internacionales donde la presencia de helmintos fue mayor $(59,3 \%$ y $63 \%)[17,18]$. En cuanto al tipo de parasitismo se encontró un predominio del poliparasitismo $(71,2 \%)$ sobre el monoparasitismo (4,6\%). La frecuencia de tres o más especies de parásitos intestinales en los escolares en este estudio fue similar a lo reportado en otra investigación realizada en el municipio de Tuta, Boyacá, Colombia $(71,2 \%$ frente a $84 \%$ ) [4], fenómeno que se explica 
Tabla 3. Factores de riesgo para parásitos intestinales en escolares de una institución educativa rural de Tunja, Colombia

\begin{tabular}{lrrrrr}
\hline Factor de riesgo & \multicolumn{1}{c}{ Parasitismo } & Chi2 & Valor $p$ \\
& Ausente & Presente & Total & \\
& N. ${ }^{\circ} \%$ & N. & $\%$ & \\
\hline
\end{tabular}

Agua de consumo diario

Pozo

Acueducto

$\begin{array}{lll}5 & 5,6 & 10 \\ 18 & 20,2 & 56\end{array}$

$11,2 \quad 15$

16,9

0,528

0,467

Hierve el agua de consumo

Sí

No

Lavado de manos

Sí

No

Lavado de frutas y verduras

$$
\text { Sí }
$$

No

Camina descalzo

Sí

No

Juega con tierra

Sí

No

Contacto con animales

\section{Sí}

No

Presencia de sanitario

Sí

No

Material del piso de la vivienda

$\begin{array}{lllllllll}\text { Cerámica } & 15 & 16,9 & 32 & 36,0 & 47 & 52,8 & 2,355 & 0,521 \\ \text { Concreto } & 7 & 7,9 & 27 & 30,3 & 34 & 38,2 & & \\ \text { Tierra } & 0 & 0,0 & 2 & 2,2 & 2 & 2,2 & & \\ \text { Madera } & 1 & 1,1 & 5 & 5,6 & 6 & 6,7 & & \\ \text { Servicios públicos } & & & & & & & & 0,252 \\ \text { Ninguno } & 0 & 0,0 & 3 & 2,2 & 3 & 3,4 & 1,310 & \\ \text { Agua } & 23 & 25,8 & 63 & 70,8 & 86 & 96,6 & & \\ \text { Disposición de aguas } & & & & & & & & \\ \text { Al aire libre } & 15 & 16,9 & 53 & 59,6 & 68 & 76,4 & 2,992 & 0,082 \\ \text { Fosas } & 8 & 9,0 & 13 & 14,6 & 21 & 23,6 & & \end{array}$

$\begin{array}{llllllll}15 & 16,9 & 50 & 56,2 & 65 & 73,0 & 0,962 & 0,326 \\ 8 & 9,0 & 16 & 18,0 & 24 & 27,0 & & \end{array}$

$\begin{array}{llllllll}14 & 15,7 & 52 & 58,4 & 66 & 74,2 & 2,857 & 0,090 \\ 9 & 10,1 & 14 & 15,7 & 23 & 25,8 & & \end{array}$

$\begin{array}{llllllll}18 & 20,2 & 55 & 61,8 & 73 & 82,0 & 0,289 & 0,585 \\ 5 & 5,6 & 11 & 12,4 & 16 & 18,0 & & \end{array}$

$\begin{array}{llllllll}13 & 14,6 & 35 & 39,3 & 48 & 53,9 & 0,084 & 0,771 \\ 10 & 11,2 & 31 & 34,8 & 41 & 46,1 & & \end{array}$

$\begin{array}{llllllll}16 & 18,0 & 34 & 38,2 & 50 & 56,2 & 2,257 & 0,133 \\ 7 & 7,9 & 32 & 36,0 & 39 & 43,8 & & \end{array}$

$\begin{array}{llllllll}18 & 20,2 & 55 & 61,8 & 73 & 82,0 & 0,265 & 0,606 \\ 5 & 5,6 & 11 & 12,4 & 16 & 18,0 & & \end{array}$

$5,6,11,12,4,16,18,0$

$\begin{array}{llllllll}21 & 23,6 & 65 & 73,0 & 86 & 96,6 & 2,700 & 0,100 \\ 2 & 2,2 & 1 & 1,1 & 3 & 3,4 & & \end{array}$




\begin{tabular}{|c|c|c|c|c|c|c|c|c|}
\hline \multicolumn{9}{|l|}{ Disposición de basuras } \\
\hline Empresa recolectora & 0 & 0,0 & 11 & 12,4 & 11 & 12,3 & 5,091 & 0,165 \\
\hline Al aire libre & 8 & 9,0 & 25 & 28,1 & 33 & 37,1 & & \\
\hline Quema & 12 & 13,5 & 30 & 33,7 & 42 & 47,2 & & \\
\hline Reciclaje & 0 & 0,0 & 3 & 3,4 & 3 & 3,4 & & \\
\hline \multicolumn{9}{|c|}{ Acude a servicios médicos } \\
\hline Sí & 19 & 21,3 & 45 & 50,6 & 64 & 71,9 & 1,757 & 0,184 \\
\hline No & 4 & 4,5 & 21 & 23,6 & 25 & 28,1 & & \\
\hline \multicolumn{9}{|l|}{ Medicación en casa } \\
\hline Sí & 21 & 23,6 & 61 & 68,5 & 82 & 92,1 & 0,030 & 0,862 \\
\hline No & 2 & 2,2 & 5 & 5,6 & 7 & 7,9 & & \\
\hline
\end{tabular}

como parte de la interacción comensal/patógeno a nivel intestinal en una misma persona, que permite que distintas especies de parásitos, patógenas o no, puedan convivir en el mismo hospedero [9].

Entre los protozoos patógenos encontrados Entamoeba histolytica/dispar ocupó el primer lugar $(90,9 \%)$, aunque en un porcentaje mayor al encontrado en un estudio realizado en India, en el que también lo ubican en el primer lugar pero en el 55,3\% de los casos [19]. Por el contrario, otros estudios encontraron a esta especie parasitaria en la segunda posición de frecuencia (4,8\%) [20]. Entamoeba histolytica/dispar presenta una distribución cosmopolita, mayor en los trópicos y bajo condiciones sociosanitarias deficientes $[5,6,10]$; es causante de la amibiasis intestinal y extraintestinal, condiciones que pueden agravarse si no se diagnostican y tratan de manera adecuada y eficaz. Además, esta parasitosis persiste aun cuando ha sido continuamente estudiada y se ha destacado su rol patogénico debido a que su principal vía de transmisión es la fecal-oral mediante la ingesta de agua y alimentos contaminados con las formas infectantes $[16,21]$.

Blastocystis spp. continúa siendo un parásito con alta prevalencia como se encontró en este estudio (30,3\%). Este parásito fue considerado inicialmente como un comensal; sin embargo, estudios epidemiológicos actuales sugieren que es un patógeno que se encuentra asociado a una amplia gama de trastornos gastrointestinales y extraintestinales, y que su patogenicidad depende del número de parásitos presentes en las muestras de heces, la presencia de otros microorganismos contaminantes y el estado inmunológico del paciente. Los casos de infección humana por Blastocystis spp. se presentan en todo el mundo, pero sobre todo en los países en vías de desarrollo $[22,23]$.

En tercer lugar de los protozoos patógenos se encontró a Giardia intestinalis (6,0\%), resultado que coincide con un estudio a nivel internacional donde los porcentajes también fueron bajos (3,3\%) [24] y difiere de uno realizado en la isla Gran Canaria (España) con un mayor número de niños, pero no de zona rural, donde la prevalencia fue del 84\% mediante la detección antigénica de Gs65 en heces para el diagnóstico del parásito, lo que hace que sea precisa y aumente el número de casos positivos respecto a los métodos con los que se requiere la visualización del parásito por microscopía, bien sea por coprológico directo o por métodos 
de concentración [12]. Giardia intestinalis se ha considerado un agente de transmisión hídrica; además, es común en escuelas y centros de cuidado público, donde la infección puede seguir la ruta fecal-oral directa. De esta manera, los niños que no controlan esfínteres o que tienen malos hábitos de higiene pueden ser diseminadores de quistes de Giardia intestinalis por el contacto persona-persona. Este parásito también se relaciona con el fecalismo al aire libre, la falta de higiene y la presencia en países subdesarrollados [9].

Entre los protozoos comensales predominó Endolimax nana $(66,7 \%)$ seguida por Entamoeba coli $(60,6 \%)$, amebas no patógenas que encontradas en los escolares son indicativas de malos hábitos higiénicos, e lodamoeba butschilii (10,6\%), mientras que la menor frecuencia de especies de protozoos detectados correspondió a Chilomastix mesnilii (1,5\%). Esta predominancia y variedad de protozoos comensales encontrados en las muestras de la población estudiada carecen de importancia clínica, pero sí lo tienen a nivel epidemiológico, ya que su presencia indica contaminación fecal de los alimentos, agua ingerida con residuos fecales, agua no potable y deficiente higiene personal [16].

Con respecto a los helmintos, el que ocupó el primer lugar fue Ascaris lumbricoides $(15,1 \%)$, similar al estudio realizado por Jacinto y colaboradores (2012), donde se encontró en un $16,9 \%$ [25], lo que se puede justificar en que para la transmisión de esta especie parasitaria desempeñan un papel fundamental las condiciones socioeconómicas y el medio ambiente, y que su difusión está vinculada con la contaminación fecal de la tierra y el inadecuado saneamiento ambiental [26]. Así mismo, Niyizurugero y colaboradores (2013) [27] ubican a esta especie parasitaria en el tercer puesto de frecuencia en su investigación realizada en escolares de la ciudad de Kigali, en el este de África.

En segundo nivel de preponderancia dentro de los helmintos se ubicaron las uncinarias $(4,5 \%)$, dato que es similar al encontrado en investigaciones internacionales $(0,3 \%$ y $3,12 \%)[19,28]$ y difiere de las investigaciones realizadas a nivel nacional, en las que se ha encontrado en proporciones más altas $(34,1 \%)$ [8]. El helminto menos encontrado fue Trichuris trichiura (1,5\%), pero es importante tener en cuenta que las infecciones por este parásito producen en los niños alteraciones en la mucosa intestinal, disminución del consumo de alimentos, deficiencia de hierro y pérdida de proteínas, por lo cual se debe hacer un seguimiento a los escolares con este tipo de parasitosis para evitar el avance de las manifestaciones y poder controlarlo a tiempo [29].

Es importante resaltar que los niños sufren las consecuencias de las enfermedades infecciosas, como en el caso del parasitismo intestinal que limita el desarrollo físico y mental, y se pueden asociar a anemia y desnutrición, por lo que se debe prestar particular importancia para detectar a tiempo este problema de salud púbica en la población escolar [29]. En este estudio se identificaron algunos factores de riesgo importantes en la cadena epidemiológica de las infecciones parasitarias; aunque no hubo una relación estadísticamente significativa con la prevalencia de los parásitos intestinales se debe prestar atención a estas condiciones ya que se encontraron en altos porcentajes, entre los que se destacan la medicación en casa $(92,1 \%)$, el contacto con animales $(82,0 \%)$ y la disposición de aguas residuales al aire libre $(76,4 \%)$. 
Caminar descalzos y jugar con tierra permite adquirir parásitos que en sus ciclos de vida necesariamente pasan por allí para alcanzar su maduración [10]. Aunque en este estudio no se tuvo una alta prevalencia de helmintos, dichas prácticas se deben evitar al máximo ya que en las comunidades rurales rara vez se acude al médico por control y los ciclos biológicos parasitarios dentro del hospedero pueden avanzar ocasionando sintomatologías graves y consecuencias fatales en los niños [17]. El no lavado de las frutas y verduras antes de consumirlas permite que las formas parasitarias que pueden estar presentes, posiblemente por el continuo uso de aguas contaminadas para el riego de las mismas, sean ingeridas; por este motivo, se considera de gran importancia el lavado de los alimentos como medida de prevención para las parasitosis intestinales. Los hábitos higiénico-sanitarios inadecuados y una vivienda con diferentes falencias en su infraestructura son factores muy importantes a considerar en las parasitosis intestinales, pues cuando están presentes «abren las puertas» para la penetración de parásitos en el hospedador humano; aunque sea diagnosticado oportunamente y tratado, si continúa incurriendo en ellos se reinfecta e incrementa el deterioro de las diferentes funciones vitales $[18,20,30]$.

El contacto de los niños con animales domésticos, especialmente perros, gatos, vacas y cerdos debe ser de cuidado ya que pueden ser transmisores de enfermedades [10], entre las más importantes las parasitarias, mediante la contaminación ambiental y de alimentos o la transmisión directa, por lo que se deben adoptar medidas higiénicas y sanitarias con relación al contacto con los animales para evitar que deterioren la salud humana. La disposición de aguas residuales y basuras al aire libre contribuye al incremento de las enfermedades infecciosas y significa una amenaza para el ambiente, pues son sitios que se pueden convertir en criaderos de animales transmisores de enfermedades. Esta práctica es común en las áreas rurales, según lo han mostrado varios estudios $[17,30]$ y se debe evitar su realización para prevenir la persistencia y diseminación de las parasitosis intestinales y otras enfermedades infecciosas. La medicación en casa es una práctica muy antigua que ha sido utilizada desde hace varios siglos, que ha ocasionado que los ciclos biológicos de los parásitos se repitan y el parasitismo aumente en dichas poblaciones.

Como parte de esta investigación se les dio tratamiento gratuito a los escolares que lo requerían. Al comparar esta estrategia con la propuesta por la OMS, denominada "Quimioterapia Preventiva Antihelmíntica», la cual está diseñada para su operatividad en el contexto de la Atención Primaria en Salud (APS) con la desparasitación masiva como parte de las estrategias para la prevención y el control de las geohelmintiasis definidas en el Plan Decenal de Salud Pública Nacional 2012-2021 [31], en este caso se fue más allá; se les administró antiparasitarios para protozoos y helmintos dependiendo del caso en particular de cada escolar. Además, se impartieron sesiones educativas a padres de familia, niños y profesores sobre la epidemiología de las enfermedades parasitarias, educación en salud sobre los factores de riesgo identificados y medidas higiénico-sanitarias que fueron muy importantes para que las personas adquirieran conocimientos $y$ prácticas para mejorar $y$, en alguna medida, remediar el problema de la adquisición, la transmisión y la permanencia de los parásitos intestinales en sus comunidades. 


\section{Conclusiones}

La prevalencia de parásitos intestinales en los escolares estudiados fue del $74,2 \%$, predominando los protozoos y los parásitos no patógenos; además, se tuvo como hallazgo importante el poliparasitismo, con predominio de Entamoeba histolytica/dispar, Endolimax nana y Entamoeba coli. El estudio identificó los siguientes factores de riesgo de importancia en la cadena epidemiológica de las infecciones parasitarias: disposición de aguas residuales al aire libre, contacto con animales, jugar con tierra y caminar descalzos. A pesar de haber encontrado un predominio de parásitos intestinales no hubo una relación estadísticamente significativa con los factores predisponentes. Por tal razón, es importante continuar incrementando las actividades educativas encaminadas a prevenir el parasitismo intestinal.

\section{Bibliografía}

1. Lucero-Garzón TA, Álvarez-Motta LA, Chicue-López JF, López-Zapata D, Mendoza-Bergaño CA. Parasitosis intestinal y factores de riesgo en niños de los asentamientos subnormales, Florencia-Caquetá, Colombia. Rev Fac Nac Salud Pública 2015; 33: 171-180.

2. Arencibia-Sosa $\mathrm{H}$, Lobaina-Lafita JL, Terán-Guardia C, Legrá-Rodríguez R, Arencibia-Aquino A. Parasitismo intestinal en una población infantil venezolana. MEDISAN 2013; 17: 742-748.

3. Diéguez-Leiva K. Parasitismo intestinal en niños que asisten a un círculo infantil del municipio de Puerto Padre. Rev Electrón Dr Zoilo E Marinello Vidaurreta 2014; 39: 1-5.

4. Suescún-Carrero SH. Prevalencia de parásitos intestinales y factores de riesgo en escolares del colegio Chicamocha Kennedy I del municipio de Tuta - Boyacá, Colombia. Rev Univ Salud 2013; 15: 218-224.

5. Cañas-Ávila N, Fariñas-Reynoso AT, Rico-López TB, Suárez-Tamayo S, Benítez-Martínez M. Parasitismo intestinal en escolares, Parroquia Pozo Verde, Estado Bolívar, Venezuela. Septiembre-Octubre 2006. Hig Sanid Ambient 2013; 13: 975-979.

6. Mohammad KAE-A, Mohammad AAE-A, EI-Nour MFA, Saad MY, Timsah AG. The prevalence and associa- ted risk factors of intestinal parasitic infections among school children living in rural and urban communities in Damietta Governorate, Egypt Academ Arena 2012; 4: 90 97.

7. Organización Mundial de la Salud. Helmintiasis transmitidas por el suelo: Nota descriptiva. 2017. Disponible: http://www.who.int/mediacentre/factsheets/fs366/es/. Consultado: feb 2017.

8. República de Colombia, Ministerio de Salud y Protección Social. Encuesta Nacional de Parasitismo Intestinal en Población Escolar, Fase II. 2013. Disponible: www. minsalud.gov.co/sites/rid/Lists/BibliotecaDigital/RIDE/ VS/PP/ET/FASE\%20ENCUESTA\%20NAL\%20PARASIT.pdf. Consultado: feb 2017.

9. Botero D, Restrepo M. Parasitosis humanas (ed 5a). Medellín, Colombia: Corporación para Investigaciones Biológicas; 2012.

10. Rodríguez-Sáenz AY. Factores de riesgo para parasitismo intestinal en niños escolarizados de una institución educativa del municipio de Soracá - Boyacá. Rev Univ Salud 2015; 17: 112-120.

11. Carmona-Fonseca J, Correa-Botero A. Parásitos intestinales, desnutrición y condiciones de vida de niños en Urabá (Colombia): soledad y olvido. Rev Salud ambient 2013; 13: 108-119.

12. Novo-Veleiro I, Martín-Sánchez AM, Elcuaz-Romano R, Muro A, Afonso-Rodríguez O, García-Bardeci D, et al. Parasitosis en Gran Canaria (España). Estudio prospectivo multicéntrico durante un año. Rev Ibero-Latinoam Parasitol 2012; 71: 34-41.

13. Fernández-Niño JA, Reyes-Harker P, Astudillo-García Cl, Heredia RD, López MC, Moncada LI. Implementación y evaluación de una estrategia combinada de educación en salud y quimioterapia masiva para el control de las geohelmintiasis, en una zona rural de Colombia. Rev Univ Ind Santander Salud 2015; 47: 137-149.

14. Ávila-Labrada M, Usiña-Pozo M, Guerra-Pompa O, Pulgar-Rodríguez R. Intervención educativa para prevenir el parasitismo intestinal en niños de 0 a 9 años. Rev Electrón Dr Zoilo E Marinello Vidaurreta 2015; 40.

15. República de Colombia, Ministerio de Salud y Protección Social. Resolución número 8430 de 1993. 1993. Disponible: https://www.minsalud.gov.co/sites/ $\mathrm{rid} /$ Lists/BibliotecaDigital/RIDE/DE/DIJ/RESOLUCION8430-DE-1993.PDF. Consultado: feb 2017.

16. Cardona-Arias JA, Bedoya-Urrego K. Frecuencia de parásitos intestinales y evaluación de métodos para su diagnóstico en una comunidad marginal de Medellín, Colombia. latreia 2013; 26: 257-268.

17. Garaycochea O, Acosta-García G, Vigo-Ames N, Heringman $\mathrm{K}$, Dyer $\mathrm{A}$, Jerí $\mathrm{S}$, et al. Parasitismo intestinal, anemia y estado nutricional en niños de la comunidad de Yantaló, San Martín, Perú. Rev Ibero-Latinoam Parasitol 2012; 71: 143-151.

18. Abossie A, Seid M. Assessment of the prevalence of 
intestinal parasitosis and associated risk factors among primary school children in Chencha town, Southern Ethiopia. BMC Public Health 2014; 14: 166.

19. Bisht D, Verma AK, Bharadwaj HH. Intestinal parasitic infestation among children in a semi-urban Indian population. Trop Parasitol 2011; 1: 104-107.

20. Pérez-Sánchez G, Redondo-de la Fé G, Fong-Rodríguez HG, Sacerio-Cruz M, González-Beltrán O. Prevalencia de parasitismo intestinal en escolares de 6-11 año. MEDISAN 2012; 16: 551-557.

21. Batista-Rojas 0 , Álvarez-Hernández Z. Parasitismo intestinal en niñas y niños mayores de 5 años de Ciudad Bolívar. MEDISAN 2013; 17: 585-591.

22. Méndez Bustelo MA, Muiño Joga Md, Garabal Sánchez S, Ben López E, Llovo Taboada J. Blastocystis hominis, un gran desconocido. Rev Pediatr Aten Primaria 2015; 17: e39-e44.

23. Díaz Cajal MA, Varengo HT, Marini V, Osilles MA. Prevalencia de Blastocystis sp en niños y adolescentes de comunas periurbanas de la ciudad de Córdoba, Argentina. Rev Ibero-Latinoam Parasitol 2011; 70: 35-41.

24. Pensabene L, Talarico V, Concolino D, Ciliberto D, Campanozzi A, Gentile T, et al. Postinfectious functional gastrointestinal disorders in children: a multicenter prospective study. J Pediatr 2015; 166: 903-907 e901.

25. Jacinto E, Aponte E, Arrunátegui-Correa V. Prevalencia de parásitos intestinales en niños de diferentes niveles de educación del distrito de San Marcos, Ancash, Perú. Rev Med Hered 2012; 23: 235-239.
26. Mascarini-Serra L. Prevention of Soil-transmitted Helminth Infection. J Glob Infect Dis 2011; 3: 175-182.

27. Niyizurugero E, Ndayanze JB, Bernard K. Prevalence of intestinal parasitic infections and associated risk factors among Kigali Institute of Education students in Kigali, Rwanda. Trop Biomed 2013; 30: 718-726.

28. Nastasi-Miranda JA. Prevalencia de parasitosis intestinales en unidades educativas de Ciudad Bolívar, Venezuela. Rev Cuid 2015; 6: 1077-1084.

29. Oropesa-Vergara OL, Quevedo-Freites G, Leovigildo Leyva-Delgado, Ferra-García BM, Ferrer-Herrera IM, Rodríguez-Martínez N. Intervención educativa sobre parasitismo intestinal en niños de la Escuela Primaria Salvano Velazco, Bocono. CCM 2010; 14: 1-12.

30. Belo VS, Oliveira RBd, Fernandes PC, Nascimento BWL, Fernandes FV, Castro CLF, et al. Fatores associados à ocorrência de parasitoses intestinais em uma população de crianças e adolescentes. Revista Paulista de Pediatria 2012; 30: 195-201.

31. República de Colombia, Ministerio de Salud y Protección Social, Dirección de Promoción y Prevención, Subdirección de Enfermedades Transmisibles. Lineamiento de Desparasitación Antihelmíntica Masiva, en el Marco de la Estrategia «Quimioterapia Preventiva Antihelmíntica de OMS». 2013. Disponible: https:// www.minsalud.gov.co/sites/rid/Lists/BibliotecaDigital/RIDE/VS/PP/ET/LINEAMIENTO\%20DESPARASIT\%20 ANTIHELM\%C3\%8DNTICA\%20080122014.pdf. Consultado: feb 2017.

Introduction: Parasitic intestinal diseases are one of the most predominant among infectious diseases, finding distributed worldwide, especially in most vulnerable people. Objective: To determine the prevalence of intestinal parasites and the risk factors in schoolchildren of rural educational institution from Tunja (Colombia). Materials and methods: A transversal descriptive study has been carried out in 89 schoolchildren that agreed to participate with the prior written informed consent and assent from their parents. Stool samples from each child were collected and coprological test and analysis by formalin-ether concentration technique were conducted. The risk factors were assessed by a survey, which included questions about hygiene habits and housing characteristics. Results: The overall infection of intestinal parasites was $74.2 \%$ being Entamoeba histolytica/dispar (90.9\%), followed by Endolimax nana (66.7\%), and Entamoeba coli (60.6\%) the most frequent species. Risk factors include self-medication, animal contact, outdoor sewage disposing, play with soil, and walking barefoot. It was not found a significant relationship between risk factors and intestinal parasites. Conclusions: The proportion of intestinal parasites in schoolchildren was high (74.2\%), with the protozoa and nonpathogenic parasites as most predominant. It is important to continue increasing educational efforts to prevent intestinal parasites.

Keywords: Child, parasitic intestinal diseases, risk factors. 
గ Laboratorio ${ }^{\oplus}$

Clínico

U Hematológico

Creemos en los sueños de todos los colombianos que llevan en la sangre confianza

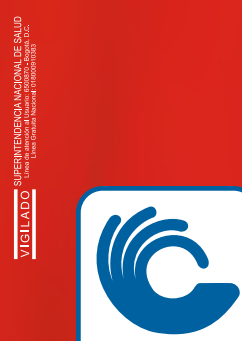

ISO 9001

4. icontec

Sede Principal Poblado: Carrera 43C No. 5-33

Toma de Muestras: Centro Comercial Sandiego, Torre Norte, Piso 10, No. 1034

Teléfono: 4444200 - www.lch.co - Medellín 